\title{
Hemorragia intracraneal como presentación de sindrome hemorragico severo por lonomismo sistemico
}

\author{
Intracranial hemorrhage as presentation of \\ severe hemorragic sindrome by sistemic \\ Ionomisms. \\ Marie Ruiz-Gonzáles /; \\ ,Johan Azañero-Haro2; \\ Andrés Alcántara-Díaz2 ;

\section{Alonso Soto 3} Ruiz-Gonzáles M. Azañero-Haro J, Alcántara-Díaz A y Soto A. Hemorragia intracraneal como presentación de sindrome hemorragico severo por lonomismo sistemico. Rev Soc Peru Med Interna. 2020;33(1):31-35.

https://doi.org/10.36393/spmi.v33i1.508

\begin{abstract}
RESUMEN
Los accidentes causados por los estadios larvarios de los lepidópteros se conocen como erucismo. El contacto accidental con las orugas del género Lonomia spp. puede desencadenar síndromes hemorrágicos que en casos severos puede llegar a producir injuria renal aguda y hemorragia intracraneal. Se presenta el caso de un varón de 48 años procedente la selva central del Perú, quien mientras estaba en el campo, incidentalmente entra en contacto con una oruga, cursando luego con un síndrome hemorrágico caracterizado por coagulopatía y hemorragia subaracnoidea. El cuadro se resolvió satisfactoriamente luego de la administración del suero antilonómico. Se discute los aspectos fisiopatológicos, clínicos y laboratoriales que condujeron al diagnóstico, así como la efectividad reportada del tratamiento.
\end{abstract}

Palabras clave: erucismo, lepidopterismo, síndrome hemorrágico, Lonomia spp.

\section{ABSTRACT}

The accidents caused by the larval stages of the Lepidoptera are known as erucism. Accidental contact with caterpillars of the genus Lonomia spp. can trigger hemorrhagic syndromes that in severe cases can lead to acute renal injury and intracranial hemorrhage. We present the case of a 48 year-old male from Peruvian Central Jungle who while was working in the forest comes in contact with a caterpillar, after which he developed an hemorrhagic syndrome characterized by coagulopathy and subarachnoid hemorrhage. The clinical picture resolved satisfactorily after administration of antilonomic serum. The pathophysiological, clinical and laboratory aspects that led to the diagnosis are discussed, as well as the reported effectiveness of treatment.

Key words: erucism, lepidopterism, hemorrhagic syndrome, Lonomia spp.

1 Médico residente de medicina interna. Universidad Federico Villarreal. Hospital Nacional Hipólito Unanue, Lima. 2 Médico internista. Departamento de Medicina, Hospital Nacional Hipólito Unanue, Lima.

3 Médico internista. Instituto de Investigación en Ciencias

Biomédicas (INICIB), Universidad Ricardo Palma. Departamento de Medicina, Hospital Nacional Hipólito Unanue.

\section{INTRODUCCIÓN}

El término lepidopterismo se utiliza de forma generalizada para describir los efectos tóxicos, locales y sistémicos, producidos en humanos por las formas adultas (mariposas, polillas) mientras que a las manifestaciones clínicas asociadas con los estados inmaduros de las mariposas se les conoce como erucismo. ${ }^{(1-3)}$ Entre los lepidópteros, las formas larvarias (orugas) son las que tienen efectos sistémicos en humanos y son consideradas de importancia clínica. ${ }^{(2,4)}$

De las más de 127 familias de mariposas y 165000 especies a nivel mundial, solo 12 familias tienen importancia clínica y epidemiológica a nivel mundial. En Latinoamérica (Brasil, Venezuela, Perú, Paraguay, Colombia, Guyana Francesa y Argentina,), la principal familia reportada como causante de erucismo es la familia Saturniidae. ${ }^{(1,5)}$

Dentro de la familia Saturniidae se incluyen 26 especies que se encuentran distribuidas en el continente americano, y solo las del género Lonomia (especies obliqua y achelous) son las que presentan los síndromes hemorrágicos graves de tipo fibrinoproteolítico por contacto con las orugas, condición conocida como lonomismo. 
L. achelous se encuentra principalmente en Venezuela, Colombia, la Guyana Francesa y existen reportes en Paraguay, Perú y Ecuador. L. obliqua se encuentra en el sur de Brasil, Uruguay y una pequeña zona del norte de Argentina. ${ }^{(1,5)}$

Por lo general, los accidentes por Lonomia spp ocurren cuando la víctima se inclina contra decenas, a veces cientos, de orugas que reposan sobre el tronco de un árbol. Al ser aplastadas, se entra en contacto con las cerdas las cuales se rompen y la secreción venenosa penetra en la piel. ${ }^{(6)}$ Los pacientes afectados en la mayoría de casos presentan dolor y ardor leve en la zona del contacto, náuseas y cefalea, pero puede progresar a un síndrome hemorrágico severo caracterizado por equimosis, hematuria, sangrado de heridas y mucosas y, en algunos casos, hemorragia intracerebral e insuficiencia renal aguda. ${ }^{(7,8)}$

En el Perú han sido reportados cuatro casos hasta la fecha, tres de ellos en personas nativas, procedentes de la selva central del país, quienes cursaron con cuadros hemorrágicos severos asociados a trastornos de la coagulación producidos inmediatamente después del contacto con orugas del género Lonomia spp., y un caso en una paciente en el noreste del país. ${ }^{(9)}$ Aunque el subregistro sigue siendo alto y el conocimiento de la temática escaso, el tema va adquiriendo importancia debido a la posible gravedad de los casos, su expansión geográfica y la disponibilidad actual de suero antilonómico, el cual puede evitar la muerte del paciente si el cuadro se diagnostica oportunamente.

\section{PRESENTACIÓN DEL CASO}

Paciente varón de 48 años de edad, procedente de la provincia de Satipo, distrito de Río Negro, Anexo San Miguel de Cobreyoque (selva central del Perú), Junín, agricultor, quien seis días antes de su ingreso, mientras realizaba sus actividades agrícolas entró en contacto el dorso de su mano izquierda con una oruga que se encontraban en el tallo de un árbol de pacay. De acuerdo a la descripción del paciente, la oruga era de color marrón claro, de aproximadamente 6 a $8 \mathrm{~cm}$ de tamaño con pilosidad de color blanco en el dorso. Al día siguiente presentó dolor y equimosis en la flexura del brazo izquierdo. Acudió al establecimiento de salud de su localidad donde se le administró sintomáticos. A los dos días aparecieron lesiones equimóticas en los miembros inferiores asociadas a astenia. Mientras realizaba actividades domiciliarias sufrió un traumatismo en la pierna derecha presentando equimosis en dicha zona y acompañándose de dolor intenso con limitación funcional por lo que acudió al hospital de Satipo donde le administraron analgésico intramuscular, presentando una equímosis en la región glútea derecha.

Tres días antes del ingreso, presentó gingivorragia autolimitándose en el mismo día, acompañándose de dolor abdominal tipo cólico, de intensidad leve, más cefalea occipital de moderada intensidad y dolor retroocular.

Un día antes de su ingreso el malestar general y cefalea se intensificaron motivo por el cual acudió al Servicio de Emergencia del Hospital Nacional Hipólito Unanue, Lima. Al ingreso, presentó presión arterial de 110/70 mm $\mathrm{Hg}$, múltiples equimosis en los miembros inferiores a predominio derecho con cambios de coloración circundantes y acompañados de aumento de volumen difuso, rubor y aumento de temperatura local que se extendían hasta la parte posterior de muslo derecho; equímosis en la región glútea derecha de aproximadamente $10 \times 7 \mathrm{~cm}$ con eritema circundante y rubor, equímosis en cara anterior y lateral interna del antebrazo izquierdo (Figura 1); dolor a la movilización y palpación profunda en los miembros inferiores. En el examen de tórax y pulmones y cardiovascular no se evidenció alteraciones. En el examen de abdomen se evidenció dolor difuso leve, sin visceromegalias. En el examen del sistema nervioso no evidenció alteraciones motoras ni sensitivas, sin signos meníngeos o de liberación piramidal. El resto de examen no fue contributorio.

Dentro de los resultados de exámenes auxiliares el hematocrito fue de $18,8 \%$, el recuento de leucocitos de $13640 / \mu \mathrm{L}$ con fórmula diferencial sin alteración 310000 plaquetas/ $\mu \mathrm{L}$, TTP 36,46 seg, TP 16,94 seg, INR 1,47, fibrinógeno $66 \mathrm{mg} / \mathrm{dl}$ (VN: 200 - $400 \mathrm{mg} / \mathrm{dl}$ ), no evidencia de hemólisis. Radiografía de tórax normal y ecografía abdominal: presencia de líquido libre en región pélvica en escasa cantidad.

Tabla 1. Evolución en el tiempo de los exámenes de laboratorio.

$\begin{array}{llllllllll}\text { Día } & 1 & 2 & 3 & 4 & 5 & 6 & 7 & 9 & 10 \\ \mathrm{Hb}(\mathrm{gr} / \mathrm{dl}) & 6,4 & 6,5 & & 7,3 & 7,1 & 7,0 & 7,8 & 8,1 & 9,4 \\ \text { Plaquetas } \times 10^{3} / \mathrm{dl} & 310 & 400 & & 450 & 485 & 494 & 534 & 600 & 700 \\ \text { TP (seg) } & 17,0 & 17,8 & 14,0 & 13,8 & 13,8 & 13,3 & 12,0 & 12,4 & 11,8 \\ \text { TTP (seg) } & 36,2 & 37,0 & 32,0 & 29,0 & 28,5 & 29,1 & 28,0 & 28,0 & 28,0 \\ \text { INR } & 1,48 & 1,55 & 1,22 & 1,17 & 1,16 & 1,12 & 0,99 & 1,03 & 0,98 \\ \text { Fibrinógeno (mg/dl) } & & 66 & 107 & 123 & 193 & 295 & 457 & 421 & 498\end{array}$




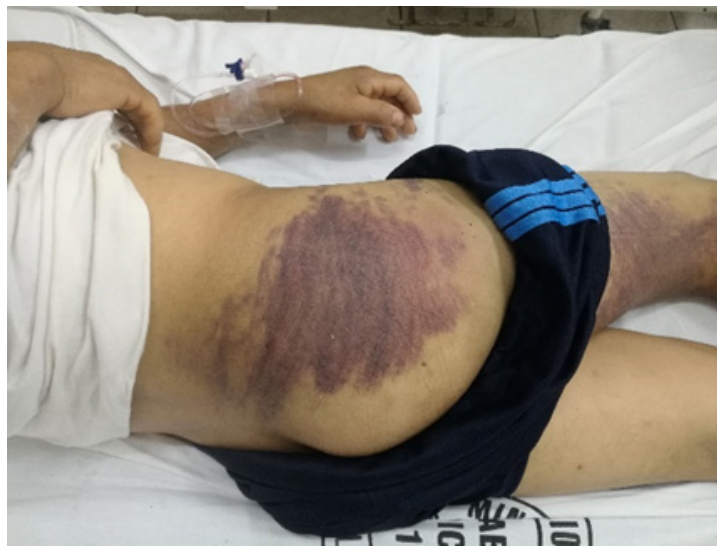

Figura I. Equimosis en piernas y región glútea derecha

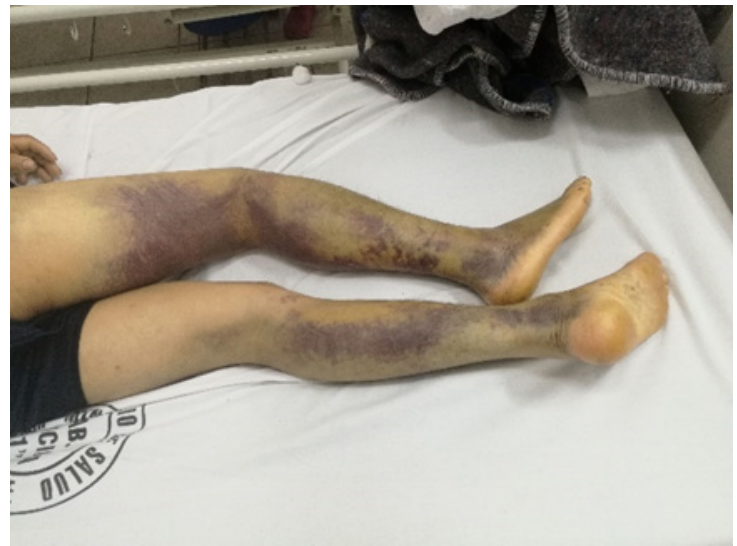

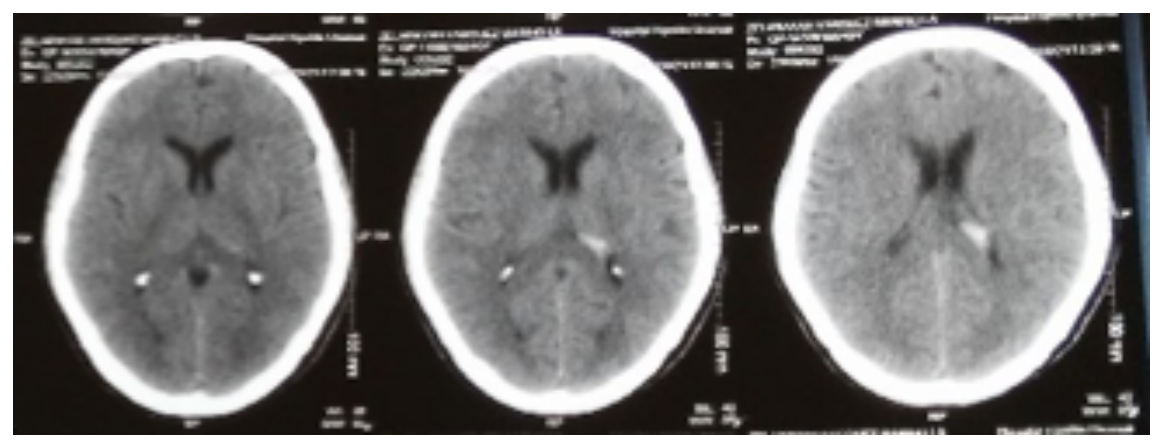

Figura 2.TAC cerebral:hemorragia subaracnoidea Fisher 4 (imagen previa a la administración de suero antilonómico).

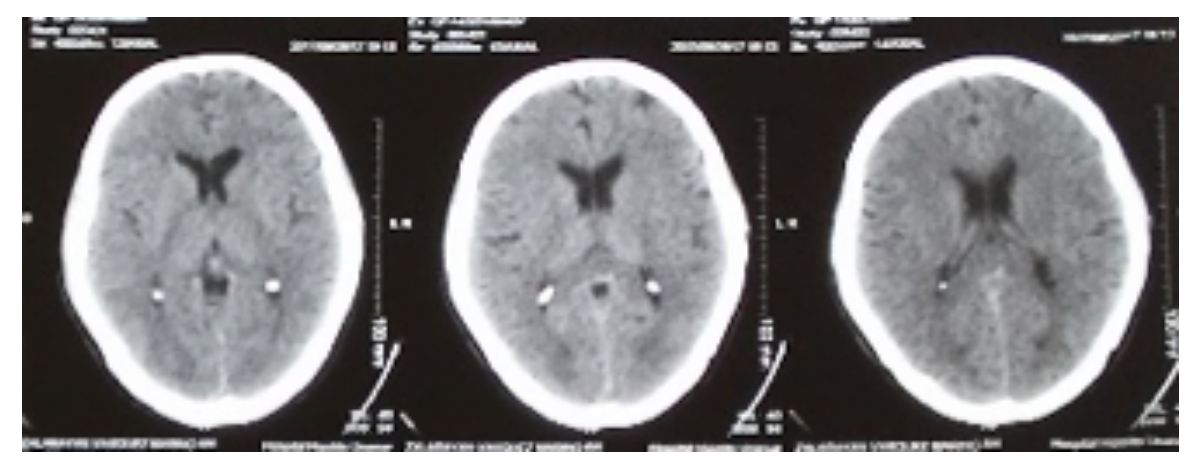

Figura 3.TAC cerebral sin contraste, 48 horas post administración de suero antilonómico.

Permaneció hemodinámicamente estable durante su hospitalización, persistiendo con cefalea pulsátil holocraneana de intensidad moderada. Al ingreso, no se contó con el suero antilonómico por lo que se le administro cuatro unidades de crioprecipitados. Por la persistencia de cefalea intensa se le realizó un TEM cerebral donde se evidenció una hemorragia subaracnoidea interhemisferica e intraventricular Fisher 4.

Se obtuvo el suero antilonómico gracias a las gestiones conjuntas con el Instituto Nacional de Salud y el Instituto Butantán de Brasil. Se le premedicó con hidrocortisona $100 \mathrm{mg}$ y clorfeniramina $10 \mathrm{mg}$ endovenoso. Se administró suero antilonómico (10 viales endovenosos). No hubo efectos adversos durante su administración. El dosaje de fibrinógeno a las 3 horas posteriores del suero fue $107 \mathrm{mg} /$ $\mathrm{dl}$ y a las 9 horas posteriores $123 \mathrm{mg} / \mathrm{dl}$. El paciente refirió que la cefalea disminuyó en intensidad. Se realizó una TEM cerebral control en 48 horas con evolución favorable en relación al anterior. Al examen físico se evidenció disminución progresiva de las equímosis y hematomas, mejoría clínica paulatina y luego del tercer día el perfil de coagulación se normalizó (Ver Tabla 1 y Figuras 1-3).

\section{DISCUSIÓN}

Este es el quinto caso reportado en el Perú de accidentes con orugas del género Lonomia spp. probablemente porque 
estos casos ocurren en áreas rurales y no son de notificación inmediata. Al contrario de lo que se ve en Brasil, donde hay alta incidencia de erucismo por Lonomia obliqua, especialmente en las regiones del sur donde es considerado un problema de salud por sus tasas morbilidad y mortalidad. (9)

De las dos especies de Lonomia que ocasionan síndrome hemorrágico, L. achelous es encontrada en Venezuela, Guyana francesa, Colombia y región amazónica de Brasil; y, L. obliqua es ubicada en el sur del Brasil y en la selva de Perú ${ }^{(1)}$.

El contacto accidental con algunas orugas del género Lonomia se conoce como lonomismo y ocasiona lesiones que van desde lesiones leves en la piel (irritación, reacciones alérgicas) hasta lesiones graves como insuficiencia renal y trastornos hemorrágicos debidos al síndrome hemorrágico que desencadenan. ${ }^{(10)}$

El veneno genera una intensa activación del sistema fibrinolítico mediante una acción procoagulante o por coagulopatía de consumo, dependiendo del tipo de especie, ya que se ha demostrado en estudios que los mecanismos de alteración en la coagulación son diferentes en L. obliqua y L. achelous. $^{(12,13)}$

En el caso de L. obliqua se han identificado dos toxinas procoagulantes: un activador del factor X, llamado losac (factor activador Stuart de L. obliqua) y un activador de la protrombina llamado lopap (proteasa activadora de protrombina de L. obliqua). El veneno provoca una forma especial de coagulación intravascular diseminada (CID) con coagulopatía de consumo, agotamiento de los factores V y XIII, así como fibrinólisis secundaria. ${ }^{(13,15)}$

El veneno de L. achelous tiene actividad tanto procoagulante como anticoagulante produciendo un síndrome fibrinolítico. Las principales toxinas son lonomin II, con actividad fibrinolítica directa; lonomin III, que es un activador de protrombina, lonomin IV, que actúa como activador del Factor $\mathrm{X}$; lonomin $\mathrm{V}$, que es una enzima proteolítica que ejerce su actividad sobre el zimógeno del Factor XIII activo y sobre sus subunidades A y $\mathrm{B}$, inactivándolos, y también tiene actividad similar a la uroquinasa (activando el plasminogeno); lonomin VI:a y lonomin VI:i, que actúan como activadores o inhibidores del Factor V; lonomin VII, con actividad similar a la calicreína y a lonomin I; $\mathrm{y}$, achelasa I y achelasa II, que tienen acción fibrinolítica similares a las de la plasmina. ${ }^{(14)}$

En general, las manifestaciones hemorrágicas aparecen horas después del contacto con las cerdas de Lonomia spp., por lo que la sospecha diagnóstica demora hasta que hay manifestaciones sistémicas tales como sangrados y falla renal aguda. ${ }^{(12)}$ Los pacientes presentan una prolongación severa de los parámetros de coagulación como el tiempo de protrombina (PT) y tiempo parcial de tromboplastina activada (aPTT), disminución en los niveles plasmáticos de fibrinógeno, factores V y XIII, precalicreína, plasminógeno, proteína $\mathrm{C}$ y alfa 2-antiplasmina, y un aumento en los niveles de complejo trombina-antitrombina, fragmento $1 \mathrm{p} 2$ a partir de la activación de protrombina y dímeros D. El factor II está disminuido en casos severos a pesar de esta intensa coagulopatía de consumo; y, el número de plaquetas parece disminuir sólo en los casos severos, siendo por lo general normal en los casos leves. ${ }^{(16)}$

En la evaluación de nuestro paciente se evidenció disminución de fibrinógeno y prolongación del PT, aPTT con recuento de plaquetas normales.

Con el cuadro clínico más la anamnesis del paciente se sospechó de erucismo por Lonomia spp.

El suero antilonómico (ALS) es el único tratamiento efectivo ya que obtiene la recuperación del fibrinógeno y la normalización de los tiempos de protrombina (TP) y tiempo parcial de tromboplastina activado (aTPT), independiente del tiempo de la administración. ${ }^{(17)}$ Este suero se produce en el Instituto Butantan de Brasil, el cual recientemente refinó la preparación de ALS mediante la producción de inmunoglobulina F (ab') 2, fragmentos capaces de neutralizar los componentes del veneno de L. obliqua, e igualmente útil en erucismo por L. achelous. ${ }^{(18)}$

Desde un punto de vista terapéutico, el ALS ha sido exitosa en restablecer los parámetros de coagulación a lo fisiológica. ${ }^{(19)}$ Antes del desarrollo del ALS en Brasil, el uso de antifibrinolíticos, como el ácido e-aminocaproico (EACA), se recomendaba oficialmente como terapia contra el envenenamiento por L. obliqua por el Ministerio de Salud de Brasil con base en la experiencia clínica con $L$. achelous en Venezuela. Aunque el uso de EACA ha sido exitoso en detener el síndrome hemorrágico por L. achelous estudios recientes no lo recomiendan para L. obliqua ya que se ha visto altas tasas de mortalidad en experimentos con ratas. ${ }^{(13,20)}$

En nuestro caso se evidenció una respuesta exitosa al suero antilonómico como se demuestra en la recuperación de los valores de fibrinógeno a las horas de aplicado el ALS. La falta de producción de este suero en nuestro país es una limitación importante. Sin embargo, en este caso la coordinación rápida con centros especializados como el Instituto Butantan a través del Instituto Nacional de Salud permitió un resultado exitoso.

Finalmente, consideramos que es importante para el médico internista el conocer la existencia de manifestaciones graves secundarias al envenenamiento por contacto con orugas a fin de poder plantear rápidamente, a través de una anamnesis apropiada, la sospecha diagnóstica y tomar acciones inmediatas para evitar complicaciones más graves como han sido reportadas. Asimismo, consideramos necesario considerar la necesidad de producción de suero antilonómico en nuestro país para esta condición desatendida.

\section{REFERENCIAS BIBLIOGRÁFICAS}

I. Gómez JP. Lepidopterismo y erucismo en Colombia. Revista Biosalud. 2014; 13(2): 59-83.

2. Pineda D,Amarillo A, Becerra J. Montenegro G. Síndrome hemorrágico por contacto con orugas del género Lonomia (Saturniidae) en Casanare, Colombia: informe de dos casos. Biomédica. 2001:21:328-32.

3. Goldman BS, Bragg BN. Caterpillar and moth bites. In: StatPearls Treasure Island (FL): StatPearls Publishing; 2019. URL disponible en: https://www.ncbi.nlm.nih.gov/books/NBK53985 I 
4. Mayence C, Mathien, et al. Lonomia caterpillar envenoming in French Guyana reversed by the Brazilian antivenom: A successful case of international cooperation for a rare but deadly tropical hazard. 2018. doi: 10.1016/j.toxicon.2018.06.068.

5. Sánchez MA, Mignone Chagas MA, Casertano SA, Cavagnaro LE, Peichoto ME.Accidentes causados por la oruga Lonomia obliqua (Walker, 1855) un problema emergente. Medicina (Buenos Aires). 20I 5; 75: 328-333 6. Spadacci-Morena et al. The urticating apparatus in the caterpillar of Lonomia obliqua (Lepidoptera: Saturniidae). In: Science Direct journal homepage: Publishing; 2016 June-. Available from: www.elsevier.com/ locate/toxicon.

7. Arocha-Piñango CL, Guerrero B. Lonomia genus caterpillar envenomation: clinical and biological aspects. Haemostasis. 200 I;31:288293.

8. Peña-Vásquez W, Vásquez-Paz $H$, Vásquez-Becerra $R$, ChiappeGonzalez A, Ñavincopa-Flores M,Ticona-Chávez E. Niña con erucismo hemorrágico por Lonomia spp. Reporte de un caso. Rev Peru Med Exp Salud Publica. 2016;33(4):8I9-23. doi: I0.17843/rpmesp.2016.334.2570

9. Diaz, J.H. The evolving global epidemiology, syndromic classification, management, and prevention of caterpillar envenoming. Am J Trop Med Hyg. 2005; 72, 347-357.

10. Gambourgi GP, Metcalf EB, Barros EJG. Acute renal failure provoked by toxin from caterpillars of the species Lonomia obliqua. Toxicon. 2006; 47:68-74.

II. Eric W. Hossler. Caterpillars and moths. Dermatologic Therapy. 2009;22:353-366.

I2. Santos JHA, Oliveira SS, Alves EC, Mendonça-da-Silva I, Sachett JAG, Tavares A, et al. Severe hemorrhagic syndrome after Lonomia Caterpillar Envenomation in the Western Brazilian Amazon: How Many More Cases Are There? Wilderness \& Environmental Medicine. 20I 7;28 (I):46-50.

13. Arocha-Pinango CL, Marval E, Guerrero B. Lonomia genus caterpillar toxins: biochemical aspects. Biochimies. 2000; 82, 937-942.
14. Taylor P, Salazar E, Barrios M, et al. Role of the inflammatory response in the hemorrhagic syndrome induced by the hemolymph of the caterpillar Lonomia achelous. Toxicon. 2016 Oct; I21:77-85. DOI: 10.1016/j.toxicon.2016.08.018.

15. Bernardi L, Pinto AFM, Mendes E, Yates JR 3rd, Lamers ML. Lonomia obliqua bristle extract modulates Racl activation, membrane dynamics and cell adhesion properties. Toxicon. 2019 Apr;162:32-39. DOI: 10.1016/j.toxicon.2019.02.019.

16. Pinto AFM, Berger M, Reck J, Terra RMS, Guimaraes JA. Lonomia obliqua venom: In vivo effects and molecular aspects associated with the hemorrhagic syndrome. Toxicon. 2010;56(7): I 103-12.

17. Dias da Silva W, Campos CM, Gonçalves LR, Sousa-e Silva MC, Higashi HG, Yamagushi IK, Kelen EM. Development of an antivenom against toxins of Lonomia obliqua caterpillars. Toxicon 1996; 34, I 045- I 049. 18. Rocha-Campos AC, Gonçalves LR, Higasshi HG, Yamagushi IK, Fernandes I, Oliveira JE, Ribela MT. Sousa-e-Silva MC, Dias da Silva W. Specific heterologous $F(a b$ ')2 antibodies revert blood incoagulability resulting from envenoming by Lonomia obliqua caterpillars. Am J Trop Med Hyg. 200 I; 64, 283-289.

19. Ricci-Silva ME,Valen RH, Leon IR, Tambourgi DV, Ramos OH, Perales J, Chudzinski-Tavassi AM. Immunochemical and proteomic technologies as tools for unravelling toxins involved in envenoming by accidental contact with Lonomia obliqua caterpillars. Toxicon. 2008; 5I, I0I7-1028.

20. Gonçalves LR, Sousa-e-Silva MC, Tomy SC, Sano-Martins IS, 2007 Efficacy of serum therapy on the treatment of rats experimentally envenomed by bristle extract of the caterpillar Lonomia obliqua: comparison with epsilon-aminocaproic acid therapy. Toxicon. 2007; 50,349-356.

\section{CORRESPONDNECIA}

marieruizgonzales@gmail.com

Fecha De recepción: 18-02-2020.

Fecha de aceptación: 20-03-2020. 